\title{
Insecticide-Treated House Screens to Reduce Infestations of Dengue Vectors
}

\author{
Pablo Manrique-Saide, Azael Che-Mendoza, \\ Josué Herrera-Bojórquez, Josué Villegas Chim, \\ Guillermo Guillermo-May, Anuar Medina-Barreiro, \\ Felipe Dzul-Manzanilla, Abdiel Martín-Park, \\ Gabriela González-Olvera, Hugo Delfín-Gonzalez, \\ Juan I. Arredondo-Jiménez, \\ Adriana E. Flores-Suarez, Norma Pavía-Ruz, \\ Catrin Huws Jones, Audrey Lenhart and \\ Gonzalo Vazquez-Prokopec
}

Additional information is available at the end of the chapter

http://dx.doi.org/10.5772/67934

\begin{abstract}
The public health importance of the endophilic mosquito Aedes aegypti increased dramatically in the recent decade, because it is the vector of dengue, chikungunya, Zika and yellow fever. The use of long-lasting insecticidal nets (LLINs) fixed on doors and windows, as insecticide-treated screening (ITS), is one innovative approach recently evaluated for Aedes control in South Mexico. From 2009 to 2014, cluster-randomised controlled trials were conducted in Acapulco and Merida. Intervention clusters received Aedes-proof houses ('Casas a prueba de Aedes') with ITS and were followed up during 2 years. Overall, results showed significant and sustained reductions on indoor adult vector densities in the treated clusters with ITS after 2 years: ca. $50 \%$ on the presence (OR $\leq 0.62, \mathrm{P}<0.05$ ) and abundance (IRR $\leq 0.58, \mathrm{P}<0.05$ ). ITS on doors and windows are 'user-friendly' tool, with high levels of acceptance, requiring little additional work or behavioural change by householders. Factors that favoured these interventions were (a) house construction, (b) high coverage achieved due to the excellent acceptance by the community and (c) collaboration of the vector control services; and only some operational complaints relating to screen fragility and the installation process. ITS is a housing improvement that should be part of the current paradigms for urban vector-borne disease control.
\end{abstract}

Keywords: Aedes aegypti, dengue, chikungunya, Zika, prevention, long-lasting insecticidal nets, insecticide-treated screens, house screening 


\section{Introduction}

Dengue remains a priority for public health authorities across the globe. The viral disease is transmitted primarily by the human-biting mosquito Aedes aegypti, which also transmits other viruses, including yellow fever, chikungunya and Zika [1-3]. Although there are several vaccine candidates for dengue, chikungunya and Zika, but none is highly effective. Therefore, preventing or reducing Dengue (DEN) and the transmission of other Aedes-borne diseases depend entirely on control of the mosquito vectors or interruption of human-vector contact.

Control of $A$. aegypti is mostly performed in the context of public health and depends on structured programmes sustained by ministries of health. Routine vector control efforts, mostly targeting the outdoor allocation of Aedes vectors (i.e. source reduction, hand-applied larvicides to artificial breeding sites and outdoor ultralow volume (ULV) adulticiding), have to date achieved only a limited success in preventing diseases [4]. Improving urban Aedes control and achieving a measurable impact on DEN transmission require a reformulation of current strategies and a stronger focus on the adult mosquitoes that actually transmit the disease, both lowering vector abundance and preventing human-vector contact $[5,6]$.

Females of $A$. aegypti mosquitoes have an endophilic behaviour (within buildings) and methods targeting these locations which have great potential for sustained impact. Methods currently accessible to reduce indoor adult vector abundance and prevent human-vector contact include essentially the control of adult vectors with insecticides (adulticides) applied by institutional programmes either as residual surface treatments or as space treatments; personal protection to reduce exposure to biting with the use of chemical products such as domestic insecticides, repellents and long-lasting insecticidal nets (LLINs) deployed as curtains or bed nets and changes to human habitation or behaviour such as installing mosquito screening on doors, windows and other entry points.

We recently tested in Mexico an innovative intervention called Aedes aegypti-proof houses ('Casas a prueba de Aedes aegypti' in Spanish), involving insecticide-treated screening (ITS) with LLINs permanently fitted to windows and doors as an innovative approach to exclude A. aegypti from houses in dengue endemic areas. Here, we present the results of the evaluation of these ITS-based interventions on the dengue vectors in South Mexico.

\section{Long-lasting insecticidal nets (LLINs) for A. aegypti control}

A LLIN is a factory-produced mosquito netting preloaded with a synthetic pyrethroid insecticide that is intended to retain its biological activity for at least 20 standard washes under laboratory conditions and 3 years of recommended use under field conditions [7]. LLINs, particularly bed nets, are among the most effective approaches for controlling mosquito-borne infections and reducing the global burden of malaria [8] but also can be effective for lymphatic filariasis, Japanese encephalitis and other arboviruses [9].

The use of LLINs is considered a highly effective, safe, affordable, low-tech, long-lasting and simple intervention with effects both at the individual (i.e. bed nets preventing the vector from blood feeding) and community levels (i.e. by reducing the vector lifespan and population). 
LLINs are expected to reduce human-vector contact and reduce their life expectancy as a physical barrier, blocking mosquitoes, and as a chemical method, irritating/deterring or eventually killing mosquitoes [10,11].

Based on the successful control demonstrated against nocturnal endophilic Anopheles spp. vectors and protective efficacy of LLINs (in the form of treated bed nets) in reducing malaria transmission [8, 12, 13], the WHO Dengue Scientific Working Group of 2006 identified the development/evaluation of LLINs as a primary global research stream [14]. However, LLINs were expected to be delivered in different approaches other than bed nets considering the diurnal activity patterns of Aedes mosquitoes.

In Haiti, LLIN bed nets showed an immediate effect on immature-based indicators and dengue transmission and extended for the following 5-12 months after their deployment [15]. Other sets of more ample studies suggested the potential of LLIN as window curtains (insecticide-treated curtains (ITCs)) to reduce dengue vector densities to low levels and potentially impact on dengue transmission. In Thailand, ITCs showed immediate effects on immature-based indicators at 6 months [16]. Combining ITCs with targeting productive breeding sites in Mexico [17], Venezuela $[17,18]$ and Guatemala [19] improved the impact on A. aegypti. In a field trial carried out in Mexico, ITC interventions did not affect the indoor adult population, but it seemed to reduce the number of DEN-infected females and the human infection prevalence in some areas [20].

While ITCs can be easily introduced within DEN endemic areas, these studies showed, as found with bed nets, that ITCs required proper handling and use by local communities to be effective. Coverage of the interventions based on ITCs typically falls dramatically over time [16, 21, 22], undoubtedly compromising efficacy throughout the community. For example, in Iquitos, Peru, a sociological study found that proper use of ITC falls dramatically over time ( $45 \%$ in the second year of deploying) [21, 22]. Particularly, at the household level, the efficacy of ITCs is compromised when curtains remain open/tied back during daytime or when all house entry points cannot be protected [16-20, 23]. In Guatemala [19] and Mexico [20], it was noted that families would remove or tie back the curtains to increase ventilation during the day, compromising the utility of the intervention as $A$. aegypti is a day-biting mosquito. A solution to this is to permanently fix the LLIN to the doors and windows in the form of a screen rather than curtains $[24,25]$.

\section{House screening}

Here, we use the term house screening to refer the use of insect screens in a house. An insect screen is basically a mesh (metal wire, fibreglass or other synthetic fibres) stretched in a frame (wood or metal) fixed on the opening of a house such as a door or a window. 'Mosquito-proofing' of houses (with insect screens) is a form of environmental management based on changes to human habitation to exclude vectors and reduce man-vector-pathogen contact including mosquitoes [26, 27].

The first published work evaluating house screening as physical method to prevent mosquitoborne diseases was reported by Celli in Italy for the control of malaria among railroad workers and their families [28]. His study showed that screening porches and chimneys resulted in significant reductions on malaria incidence ( $4 \%$ with screens vs. $92 \%$ without the intervention) [29]. 
The Italian experience led to widespread screening of houses against mosquitoes in malarious areas, not only in Italy but also around the world. Examples of house screening as a malaria control intervention include workers building the Panama Canal and rural homes in the Southern United States [30]. Nevertheless, this protective and efficacious method was largely forgotten when the primary strategy of insecticidal control with DDT (dichloro-diphenyltrichloroethane) emerged [30]. Modern studies on house screening have proven significant reductions on malaria [31-33] and described to be widely accepted by communities [34].

The integration of house screening for the control of dengue was evaluated in Vietnam in the 1990s. Nguyen et al. [35] and Igarashi [36] evaluated an intervention with permethrin nets covering all openings of houses (in addition to routine anti-Aedes health education and control measures) and reported a significant reduction (close to 100\%) in the number of houses positive for dengue vectors. Furthermore, indoor A. aegypti was undetectable levels for 7 months, while in the control group, infestation gradually increased during the epidemic season, and a positive impact in preventing DEN transmission during the epidemic season (at 6 months after intervention) was observed. Therefore, screening doors and windows have been considered with the potential to limit transmission in the most recent systematic reviews $[4,9]$.

\section{Aedes aegypti-proof houses}

The intervention called Aedes aegypti-proof houses ('Casas a prueba de Aedes aegypti') involves insecticide-treated screening (ITS) with the use of long-lasting insecticidal nets (LLINs) permanently fitted to windows and doors to exclude $A$. aegypti, vector of dengue, chikungunya and Zika (Figure 1).
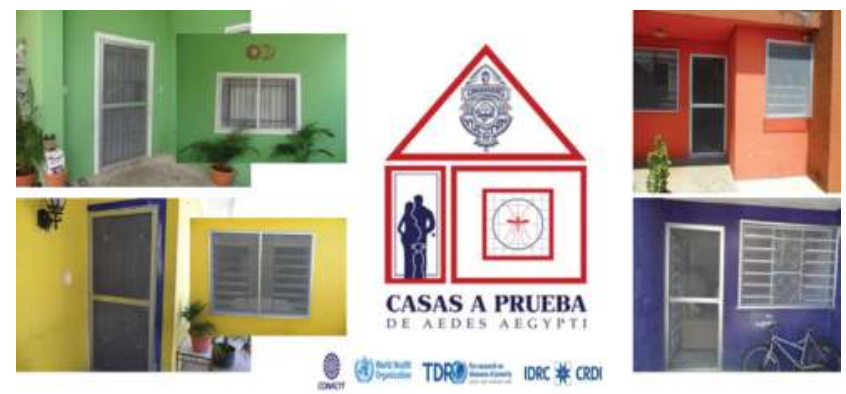

Figure 1. Photographs show Aedes aegypti-proof houses with insecticide-treated screens mounted on aluminium frames and fixed to external doors and windows of treated houses.

As described by Manrique-Saide et al. [24] and Che-Mendoza et al. [25], Duranet ${ }^{\circledR}$ screens $^{2}$ (0.55\% w.w. alpha-cypermethrin-treated non-flammable polyethylene netting [145 denier; mesh1/4132 holes/sq. inch]; Clarke Mosquito Control, Roselle, IL, USA; WHOPES approved for LLIN use) were mounted in aluminium frames custom-fitted to doors and windows of 

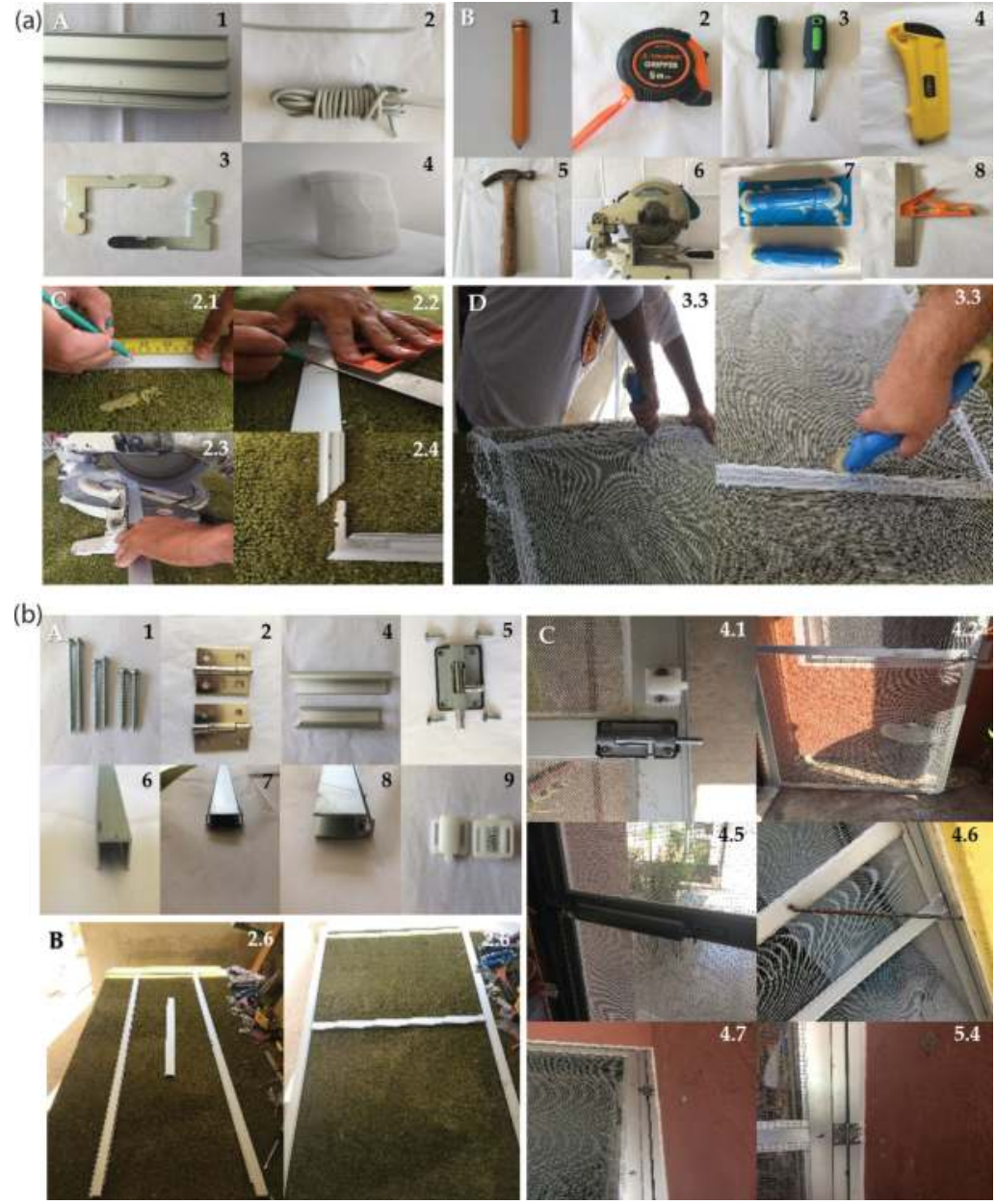

Figure 2. General description of how to install insecticide-treated screens on windows and doors. Window: (A) Materials required: (1) screen frame, (2) square for screen frame, (3) vinyl \#12 and (4) window screen. (B) Tools required: (1) pencil, (2) measuring tape, (3) screwdriver, (4) sharp knife, (5) hammer, (6) hacksaw, (7) convex wheel and (8) universal square. (C) Step 2: (2.1) Using a measuring tape and pencil, make a mark where a cut is to be made on the new frame. (2.2) With the universal square, mark a $45^{\circ}$ angle on the first mark of the final measure. (2.3) Use the hacksaw to make the $45^{\circ}$ cut. Make four cuts, one on each side of the window. (2.4) Use squares to join the four parts of the frame. Insert squares into the frame along the side with $45^{\circ}$ cut and lightly tap the squares into place. Check the assembled frame for squareness. (D) Step 3: (3.3) With the help of the convex wheel, insert the screen into the channel with vinyl, beginning at one corner (press down and roll with short strokes back and forth). When all sides have been inserted into the vinyl, remove the excess vinyl. Door: (A) Materials required: (1) screws $\left(1 \frac{1}{2} \times 8\right.$ " $\left., 1 / 2 \times 8,1 \times 8\right),(2)$ hinges, (3) vinyl \# 12, (4) elephant trunk handle, (5) hasp, (6) chair butt, (7) mosquito door frame central, (8) mosquito door frame vertical and (9) lock. (B) Step 2: (2.6) Forming the door, $1 \frac{1}{2} \times 8$ screws should preferably be used. Step 4: (4.1) The lock and pin are installed in the central part of the frame, at the appropriate distance for its correct operation. (4.2) The elephant trunk handle is installed in the central part of the frame. (4.5) Spring allows the automatic closing of the door. (4.6) Pivot allows the automatic closing of the door in a slow and controlled way. (4.7) Metal mesh protects the mosquito net from damage caused by animals. Step 5: (5.4) Fix the door to the frame using $1 / 2 \times 8$ screws, a drill and a screwdriver. Check the assembled door for squareness. 
houses in collaboration with a local small business (Figure 2). An average of two doors and five windows by house were installed in each intervention cluster. During the installation, at least one person in every household received information from research staff about the proper use and maintenance of ITS.

\section{Studies with ITS and protection against $A$. aegypti mosquitoes in Mexico}

From 2009 to 2014, cluster randomised controlled trials were conducted in two Mexican cities-Acapulco (Guerrero state) and Merida (Yucatan state) - to test the efficacy of the intervention Aedes aegypti-proof houses. The first study in Acapulco was followed by a subsequent phase of scaling-up to replication in Merida as a 'horizontal' scaling-up to expand the availability of the intervention geographically.

In general, the studies compared 10 control and 10 intervention areas of 100 households each across both cities. Routine vector control activities - as implemented by the local Ministry of Health-were performed in control clusters. Intervention clusters included insecticidetreated window and door screens (Acapulco and Merida) and targeted interventions in the productive water container types (in Acapulco only). As part of the national policy in response to dengue outbreaks and entomological risk indices [37], control and ITS intervention clusters could receive routine vector control activities (outdoor spraying with the organophosphates malathion or chlorpyrifos, indoor space spraying with the pyrethroid deltamethrin or the carbamates propoxur or bendiocarb and larviciding with the organophosphate temephos).

The main outcome metrics were the reduction of indoor vector densities. Five cross-sectional entomological surveys were conducted in intervention and control clusters as in ManriqueSaide et al. [24] and Che-Mendoza et al. [25]. Briefly, indoor adult mosquito collections were performed in a randomly selected subsample of 30 houses from each cluster (total of 300 houses per arm). A baseline survey was performed prior to the installation of ITS and was followed by surveys at 6 months of intervals corresponding to dry (low vector abundance, 6 and 18 months of post-intervention (PI)) and wet (high vector abundance, 12 and 24 of months PI) seasons. Indoor adult mosquitoes were collected with Prokopack aspirators [38] for a 15-min period per house. Collections within each cluster were performed on the same day between 09:00 and 15:00 h by skilled collector teams.

The indoor $A$. aegypti adult-based entomological indicators monitored for 2 years in both localities are shown in Figure 3. During the pre-intervention survey, similar infestation levels were quantified in both study arms. ITS protected houses against $A$. aegypti mosquitoes: significant reduction of indoor-resting adults by approximately $50 \%$ on the presence (OR $\leq$ $0.62, \mathrm{P}<0.05)$ and abundance (IRR $\leq 0.58, \mathrm{P}<0.05)$. The combination of ITS and interventions targeting productive container types was successful in continuing reducing the number of Aedes pupae and consequently of adult dengue vectors. 


\section{ACAPULCO}
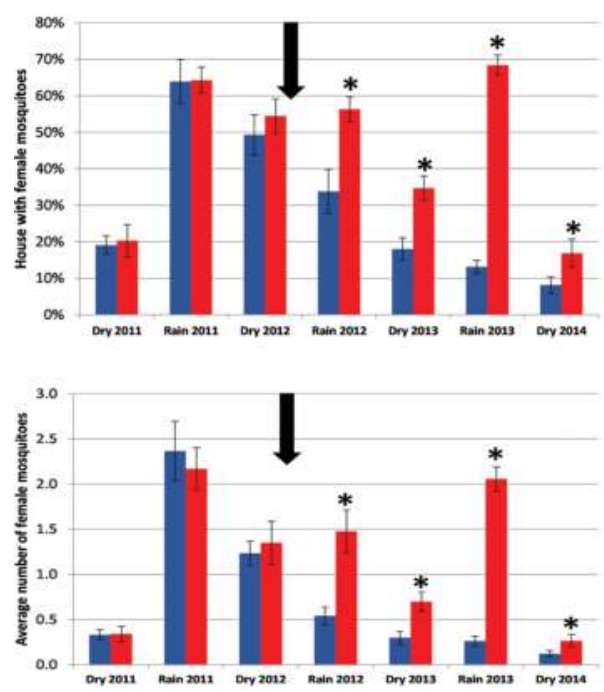

MERIDA
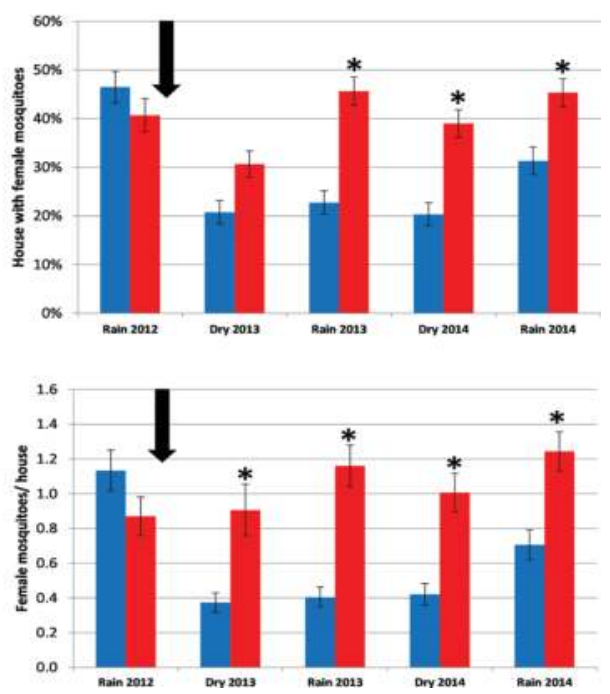

Figure 3. Comparison between groups of houses protected with insecticidetreated (blue bars) and untreated (red bars) screens on the percentage of infested houses (top) and infestation density (bottom) for female Aedes aegypti in Acapulco and Merida, Mexico. The blue arrow represents the start of the interventions. The symbol $\left({ }^{*}\right)$ denotes dates when the index was significantly different between treated and control groups on that date. Error bars show the standard error of the mean.

A significant effect on indoor adult Aedes infestations was observed in houses protected with ITS but not in controls, despite the fact that Aedes populations at both sites were resistant to pyrethroids, and this protection was sustained beyond 24 months when ITS was combined with targeted treatment of productive breeding sites (as demonstrated in Acapulco, where the combined intervention maintained a statistically significant protective effect on Aedes adult and immature stages until the end of the study, approximately 600 days after ITS installation).

Multiple factors could explain the lack of complete suppression of A. aegypti indoors by ITS. First, this method did not aim to reduce peridomestic breeding or abundance of mosquitoes. As intervention coverage on each block was not $100 \%$, there is a possibility for mosquito breeding and human feeding even in the presence of screens. In Acapulco, Mexico, combining ITS with peridomestic larval control on the most productive larval breeding habitats produced a synergistic effect evidenced as a reduction of the abundance of indoor female mosquitoes in screened houses [25]. This finding provides evidence of the importance of integrating ITS with methods focused on peridomestic control. Another factor explaining indoor presence of A. aegypti in screened houses is the fact that maybe some adults did not contact and/or survive after the initial contact with the LLIS or probably not all contacted the screened surfaces on the day when collections were performed.

Another explanation could be the loss of insecticidal power and high pyrethroid resistance in the mosquito populations of Merida. Exposure to sunlight, rain and dust impacts the residual 
power of pyrethroid insecticides. Despite of this, we demonstrated in this study that insecticidal activity of ITS under operational conditions is still acceptable after 2 years of use.

The physiological resistance in mosquitoes to the insecticide and the effect on the performance of a LLIN cannot be discounted. Pyrethroid resistance in A. aegypti populations of Mexico, including Merida, has increased during the last decade [39-42]. Currently, ITS with LLIN will have to challenge insecticide resistance to pyrethroids because they are the only insecticide class recommended and available for LLIN [43].

\section{Social acceptance and cultural barriers on Aedes aegypti-proof houses}

In Acapulco, the social studies aimed to explore the acceptance, use, adherence and perspectives of the ITS and suggestions about how to modify the programme to better address the sociocultural needs of the community. Part of the results was published by Jones et al. [44]. The main reason for acceptance was that the screens were perceived to be beneficial. The fear of violence, particularly important in Acapulco, was frequently identified as a common reason for rejection.

The most noted benefit reported for the use of ITS in Acapulco was the reduction on the amount of mosquitoes in the houses. Following screen installation, $79.9 \%$ of recipients reported that there were fewer mosquitoes inside, and a further $10.8 \%$ reported that there were none. The reduction in the amount of indoor mosquitoes was associated with a reduction in mosquito biting: $88.5 \%$ or recipients reported that mosquito biting was less of a nuisance within their homes after receiving the screens.

Participants also appreciated a reduction in mosquito numbers. Though many were unaware that the net contained insecticide, they had noticed that mosquitoes and other pests died on contact with the screen and were satisfied with this. The insecticide in the screen was seen as beneficial and acceptable, with few reports of side effects or fears about its use. Participants reported a reduction in flies and cockroaches, and $79.9 \%$ of satisfaction survey participants reported a reduction in other pests. The majority of participants $(90.2 \%)$ had the same amount or more screens in place now compared to the original amount installed, suggesting that very few were removed.

The main problem identified with the screens once installed was fragility, especially on doors. The satisfaction survey found that the most common reason for a house to have fewer screens now than originally installed was screen breakage (44\%) and higher quality material was the most frequently suggested improvement for the project. A survey of the screens found that the windows were broadly in very good condition, whereas the doors were faring less well, with $42.4 \%$ damaged in some way. Some screens were broken in exceptional accidents, but the majority of breakages reported occurred during normal use. Though some participants had feared a reduction in air circulation prior to installation, none had experienced this problem. Indeed, many expressed surprise that they had felt no effect.

Overall satisfaction with the project was very high. About $80.9 \%$ scored their satisfaction with the screens as $5 / 5,89.9 \%$ gave a score of 4 or 5 and $99.3 \%$ recommend the project to another city. 
In Merida, we conducted an ethnographic research in a small neighbourhood called Manzana 115, where 140 households are accepted to participate in the Aedes aegypti-proof houses project. In addition to an acceptance or rejection study, we also investigated 'the process of decision'. The social perception on ITS efficacy was documented since the invitation to the families. Overall, $80 \%$ of families accepted the intervention in the first invitation, whereas $30 \%$ rejected it, and at least $20 \%$ decided to accept before some family consultation. The most common reasons for acceptance were (a) that the screens were perceived to be beneficial, (b) free of cost and no future charges, (c) expectation of future 'support' and (d) high-risk perception about dengue. In Merida, the most important reasons for reluctance and rejection were (a) no interest at all; (b) low perception about dengue risk infection and (c) wives did not accept the intervention because their husbands didn't authorise it. Therefore, it is important to consider the gender roles on the decision-making processes, suggesting that both men and women must be included on social understanding about innovations for family health care.

Afterwards the installation of ITS, $80 \%$ of households reported to be very satisfied and considered the intervention effective on reducing and killing mosquitoes, not toxic for humans and pets, and also considered it important because there was no temperature increase in their houses.

\section{Conclusions}

In the absence of effective treatment or vaccines and in the context of multiple co-circulating viruses transmitted by Aedes mosquitoes, the development of preventive and long-lasting methods for Aedes control has become a top global health priority.

The benefits of house screening, as a physical barrier, rely on its efficacy to exclude mosquitoes and eventually protect against mosquito bites, which is epidemiologically relevant if most transmission occurs indoors. From an environmental health perspective, residential premises (house and peridomicile) offer important habitats for supporting populations of A. aegypti as they emerge from productive breeding sites, move in and out houses in search of food (human blood), refuge and mating and oviposit at the suitable breeding sites to complete their life cycle. Aedes is an anthropophilic, endophilic and endophagic species, and the house is the epidemiologically most significant point of vector-human contact for arbovirus transmission.

The adaptation of long-lasting insecticide nets permanently fitted as mosquito screens on windows and doors has advantages over other approaches (such as bed nets and curtains) because these interventions are in place permanently and require little additional work or behavioural change by householders.

Our studies in Mexico demonstrate that LLIS deployed as ITS acts as a barrier and significantly restrains the entrance of mosquitoes to houses for at least 2 years post deployment. Concisely, a house protected with ITS on doors and windows has at least 50\% less chances of having A. aegypti females in comparison with a non-protected house. ITS confirmed a sustained protective effect on indoor female mosquitoes, the most epidemiologically important target for vector control, even in the presence of high levels of resistance to pyrethroids in the local mosquito population. 
The present studies provide valuable and unique information on the use of house screening within cities endemic for mosquito-borne diseases, and at the time of writing, are unique in supporting the feasibility and potential benefit of this method for the simultaneous prevention and control of dengue, chikungunya and Zika transmission. The positive results from trials using house screening/full screening of windows/doors suggest that excluding the vector A. aegypti from the home may prove to be an innovative approach in terms of environmental management (changes to human habitation), if it is proven ultimately to reduce transmission of the pathogens to humans. This simple classic method of vector control should be considered and encouraged by the National Ministries of Health.

As most human-mosquito contacts occur indoors, the observed reductions in abundance and prevalence of infestation in our studies may be significant enough to impact virus transmission in a measurable way. Assessing the epidemiological impact of existing and new paradigms on Aedes-borne disease transmission remains a top research and public health priority. Particularly for ITS, our entomological studies provide evidence for the development of robust RCT (Randomized Controlled Trials) evaluating the short- and long-term protective efficacy of this method. Of particular interest is the evaluation of the potential for ITS to be included within an IVM (Integrated Vector Management) scheme that also includes other modes of vector control that target peridomestic breeding or adult abundance (e.g. larviciding, ULV).

The authorities of Mexico are considering how to expand Aedes aegypti-proof housing to as many homes as possible, probably as a targeted intervention for high-risk areas (hot spots) of endemic localities. Given that the same mosquito transmits dengue, chikungunya and Zika, the local government of Yucatan is planning to scale this intervention to protect the most vulnerable, particularly pregnant women and their families. Therefore, and in accordance with the local $\mathrm{MoH}$, our team is currently also developing a pilot study evaluating an integrated intervention on pregnant women, providing them with information and a kit of tools including repellents and Aedes aegypti-proof houses to protect them against mosquito biting and disease transmission.

Ultimately, ITS is a housing improvement that should be part of the current paradigms for urban vector-borne disease control [45].

\section{Acknowledgements}

The studies were funded by the Consejo Nacional de Ciencia y Tecnologia (Project Salud2010-01-161551), Fondo Sectorial de Investigación en Salud y Seguridad Social (SSA/IMSS/ ISSSTE-CONACYT Mexico), Fomix CONACYT-Guerrero (Project GUE-2008-02-108686), the Special Programme for Research and Training in Tropical Diseases (TDR) at the World Health Organization and the International Development Research Centre (IDRC) of Canada within the programme 'Towards Improved Dengue and Chagas Disease Control through Innovative Ecosystem Management and Community-Directed Interventions: An Eco-Bio-Social Research Programme on Dengue and Chagas Disease in Latin America and the Caribbean' (Project Number 104951-001). The nets employed in this study were donated by the company Public Health Supply and Equipment de Mexico, S.A. de C.V. Thanks to Suzanna Shugert for grammatical revision. 


\section{Author details}

Pablo Manrique-Saide ${ }^{1 *}$, Azael Che-Mendoza ${ }^{1}$, Josué Herrera-Bojórquez ${ }^{1}$, Josué Villegas Chim ${ }^{1}$, Guillermo Guillermo-May ${ }^{1}$, Anuar Medina-Barreiro ${ }^{1}$, Felipe Dzul-Manzanilla ${ }^{1}$, Abdiel

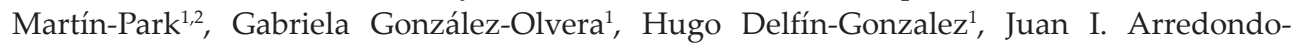
Jiménez ${ }^{3}$, Adriana E. Flores-Suarez ${ }^{3}$, Norma Pavía-Ruz ${ }^{1}$, Catrin Huws Jones ${ }^{4}$, Audrey Lenhart ${ }^{4}$ and Gonzalo Vazquez-Prokopec ${ }^{5,6}$

*Address all correspondence to: msaide@correo.uady.mx

1 Autonomous University of Yucatan, Merida, Yucatan, Mexico

2 Catedras-CONACYT, Autonomous University of Yucatan, Merida, Yucatan, Mexico

3 Autonomous University of Nuevo Leon, San Nicolas de los Garza, N.L., Mexico

4 Liverpool School of Tropical Medicine, Liverpool, UK

5 Centers for Disease Control and Prevention, Atlanta, GA, USA

6 Emory University, Atlanta, GA, USA

\section{References}

[1] Bosio CF, Fulton RE, Salasek ML, Beaty BJ, Black WC. Quantitative trait loci that control vector competence for dengue-2 virus in the mosquito Aedes aegypti. Genetics. 2000; 156: 687-698

[2] Bhatt S, Gething PW, Brady OJ, Messina JP, Farlow AW, Moyes CL, Drake JM, Brownstein JS, Hoen AG, Sankoh O, Myers MF, George DB, Jaenisch T, Wint GR, Simmons CP, Scott TW, Farrar JJ, Hay SI. The global distribution and burden of dengue. Nature. 2013; 496: 504-507. DOI: 10.1038/nature12060

[3] Li MI, Wong PS, Ng LC, Tan CH. Oral susceptibility of Singapore Aedes (Stegomyia) aegypti (Linnaeus) to Zika virus. PLoS Neglected Tropical Diseases. 2012; 6(8): e1792. DOI: 10.1371/journal.pntd.0001792. pmid: 22953014

[4] Bowman LR, Donegan S, McCall PJ. Is Dengue Vector Control Deficient in Effectiveness or Evidence?: Systematic Review and Meta-analysis. PLoS Neglected Tropical Diseases. 2016; 10(3): e0004551. DOI: 10.1371/journal.pntd.0004551

[5] Morrison A, Zielinski-Gutierrez E, Scott T, Rosenberg R. Defining challenges and proposing solutions for control of the virus vector Aedes aegypti. PLoS Medicine. 2008; 5(3): e68. DOI: 10.1371/journal.pmed.0050068

[6] Achee NL, Gould F, Perkins TA, Reiner RC, Jr., Morrison AC, Ritchie SA, Gubler DJ, Teyssou R, Scott TW. A critical assessment of vector control for dengue prevention. PLoS Neglected Tropical Diseases. 2015; 9: e0003655. DOI: 10.1371/journal.pmed.0050068 
[7] World Health Organization. Guidelines for Laboratory and Field Testing of LongLasting Insecticidal Nets. Geneva: WHO. 2013; WHO/HTM/NTD/WHOPES/2013.1

[8] Lengeler C. Insecticide-treated bed nets and curtains for preventing malaria. Cochrane Database of Systematic Reviews. 2004; 2(2): 1-54. Art. No.: CD000363. DOI: 10.1002/14651858. CD000363.pub2

[9] Wilson AL, Dhiman RC, Kitron U, Scott TW, van den Berg H, Lindsay SW. Benefit of insecticide-treated nets, curtains and screening on vector borne diseases, excluding malaria: a systematic review and meta-analysis. PLoS Neglected Tropical Diseases. 2014; 8(10): e3228. DOI: 10.1371/journal.pntd.0003228

[10] Takken W. Do insecticide-treated bednets have an effect on malaria vectors? Tropical Medicine and International Health. 2002; 7(12): 1022-1030. DOI: 10.1046/j.1365-3156. 2002.00983.x

[11] Vanlerberghe V, Villegas E, Oviedo M, Baly A, Lenhart A, McCall PJ, Van der Stuyft P. Evaluation of the effectiveness of insecticide treated materials for household level dengue vector control. PLoS Neglected Tropical Diseases. 2011a; 5(3): e994. DOI: 10.1371/ journal.pntd.0000994

[12] World Health Organization. Malaria Control Today: Current WHO Recommendations. Working document Roll Back Malaria Department. 2005a. Geneva: WHO. 75 p.

[13] World Health Organization. Insecticidetreated mosquito nets: a WHO position statement. [Internet]. 2005. Available from: http://www.who.int/malaria/publications/atoz/ itnspospaperfinal.pdf. [Accessed 2016/12/07]

[14] McCall PJ, Kittayapong P. Control of Dengue Vectors: Tools and Strategies. Report of the Scientific Working Group on Dengue. 2006. World Health Organization on behalf of the Special Programme for Research and Training in Tropical Diseases. [Internet]. 2007. pp. 110-119. Available from: http://www.who.int/tdr/publications/documents/swg_dengue_2.pdf. [Accessed 2016/10/21]

[15] Lenhart A, Orelus N, Maskill R, Alexander N, Streit T, McCall PJ. Insecticide-treated bednets to control dengue vectors: preliminary evidence from a controlled trial in Haiti. Tropical Medicine \& International Health. 2008; 13(1): 56-67. DOI: 10.1111/j.1365-3156. 2007.01966.x

[16] Vanlerberghe V, Trongtokit $Y$, Jirarojwatana S, Jirarojwatana R, Lenhart A, A piwathnasorn C, McCall PJ, Van der Stuyft P. Coverage-dependent effect of insecticide-treated curtains for dengue control in Thailand. The American Journal of Tropical Medicine and Hygiene. 2013; 89(1): 93-98. DOI: 10.4269/ajtmh.13-0015

[17] Kroeger A, Lenhart A, Ochoa M, Villegas E, Levy M, Alexander N. Effective control of dengue vectors with curtains and water container covers treated with insecticide in Mexico and Venezuela: cluster randomised trials. BMJ. 2006; 332: 1247-1252. DOI: http:// dx.doi.org/10.1136/bmj.332.7552.1247 
[18] Vanlerberghe V, Villegas E, Jirarojwatana S, Santana N, Trongtorkit Y, Jirarojwatana R, Srisupap W, Lefèvre P, Van der Stuyft P. Determinants of uptake, short term and continued use of insecticide-treated curtains and jar covers for dengue control. The American Journal of Tropical Medicine and Hygiene. 2011b; 16: 162-173. DOI: 10.1111/j.1365-3156.2010.02668.x

[19] Rizzo N, Gramajo R, Cabrera-Escobar M, Arana B, Kroeger A, Manrique-Saide P, Petzold M. Dengue vector management using insecticide treated materials and targeted interventions on productive breeding-sites in Guatemala. BMC Public Health. 2012; 12: 931-948. DOI: 10.1186/1471-2458-12-931

[20] Loroño-Pino MA, García-Rejón JE, Machain-Williams C, Gomez-Carro S, Nuñez-Ayala G, Nájera-Vázquez Mdel R, Losoya A, Aguilar L, Saavedra-Rodriguez K, LozanoFuentes S, Beaty MK, Black WC 4th, Keefe TJ, Eisen L, Beaty BJ. Towards a Casa Segura: a consumer product study of the effect of insecticide-treated curtains on Aedes aegypti and dengue virus infections in the home. The American Journal of Tropical Medicine and Hygiene. 2013; 89: 385-397. DOI: 10.4269/ajtmh.12-0772

[21] Paz-Soldan VA, Bauer KM, Lenhart A, Cordova Lopez JJ, Elder JP, Scott T, McCall PJ, Kochel TJ, Morrison AC. Experiences with insecticide-treated curtains: a qualitative study in Iquitos, Peru. BMC Public Health. 2016a; 16: 582. DOI: 10.1186/s12889-016-3191-x

[22] Paz-Soldan VA, Bauer K, Morrison AC, Cordova Lopez JJ, Izumi K, Scott TW, Elder JP, Alexander N, Halsey ES, McCall PJ, Lenhart A. Factors associated with correct and consistent insecticide treated curtain use in Iquitos, Peru. PLoS Neglected Tropical Diseases. 2016b; 10(3): e0004409. DOI: 10.1371/journal.pntd.0004409

[23] Lenhart A, Trongtokit Y, Alexander N, Apiwathnasorn C, Satimai W, Vanlerberghe V, Van der Stuyft P, McCall PJ. A cluster-randomized trial of insecticide-treated curtains for dengue vector control in Thailand. The American Journal of Tropical Medicine and Hygiene. 2013; 88: 254-259. DOI: 10.4269/ajtmh.2012.12-0423

[24] Manrique-Saide P, Che-Mendoza A, Barrera-Pérez M, Guillermo-May G, HerreraBojórquez J, Dzul-Manzanilla F, Gutierrez-Castro C, Lenhart A, Vazquez-Prokopek G, Sommerfeld J, McCall PJ, Kroeger A, Arredondo-Jiménez JI. Long-lasting insecticide treated house screens reduce domestic infestations of dengue vectors in Mexico. Emerging Infectious Diseases. 2015; 21: 308-311. DOI: 10.3201/eid2102.140533

[25] Che-Mendoza A, Guillermo-May G, Herrera-Bojórquez J, Barrera-Pérez M, DzulManzanilla F, Gutierrez-Castroc C, Arredondo-Jiménez JI, Sánchez-Tejeda G, VazquezProkopec G, Ranson H, Lenhart H, Sommerfeld J, McCall PJ, Kroeger A, Manrique-Saide P. Long-lasting insecticide treated house screens and targeted treatment of productive breeding sites for dengue vector control in Acapulco, Mexico. Transactions of the Royal Society of Tropical Medicine and Hygiene. 2015; 109: 106-115. DOI: 10.1093/trstmh/tru189

[26] World Health Organization. Manual on environmental management for mosquito control, with special emphasis on malaria vectors. 1982. WHO Offset Publication, vol. 66. Geneva 
[27] Schofield CJ, Briceno-Leon R, Kolstrup N, Webb DJT, White GB. The role of house design in limiting vector-borne disease. In: Curtis CE editor. Appropriate Technology in Vector Control. CRC Press; 1990. 233 p.

[28] Celli A. La nuova profilassi della malaria nel Lazio [The new prophylaxis against malaria in Lazio]. Supplemento al Policlinico. 1900; 6: 1601-1606

[29] Ferroni E, Jefferson T, Gachelin G. Angelo Celli and research on the prevention of malaria in Italy a century ago. Journal of the Royal Society of Medicine. 2012; 105: 35-40. DOI: 10.1258/jrsm.2011.11k049

[30] Lindsay SW, Emerson PM, Charlwood JD. Reducing malaria by mosquito-proofing houses. Trends in Parasitology. 2002; 18: 510-514. DOI: http://dx.doi.org/10.1016/S14714922(02)02382-6

[31] Kirby M, Green C, Milligan P, Sismanidis C, Jasseh M, Conway D, Lindsay S. Risk factors for house-entry by malaria vectors in a rural town and satellite villages in The Gambia. Malaria Journal. 2008; 7: 2. DOI: 10.1186/1475-2875-7-2

[32] Kirby MJ, Ameh D, Bottomley C, Green C, Jawara M, Milligan PJ, Snell PC, Conway DJ, Lindsay SW. Effect of two different house screening interventions on exposure to malaria vectors and on anaemia in children in The Gambia: a randomised controlled trial. Lancet. 2009; 374: 998-1009. DOI: 10.1016/S0140-6736(09)60871-0

[33] Lindsay SW, Jawara M, Paine K, Pinder M, Walraven G, Emerson PM. Changes in house design reduce exposure to malaria mosquitoes. Tropical Medicine \& International Health. 2003; 8: 512-517. DOI: 10.1046/j.1365-3156.2003.01059.x

[34] Kirby MJ, Bah P, Jones CO, Kelly AH, Jasseh M, Lindsay SW. Social acceptability and durability of two different house screening interventions against exposure to malaria vectors, Plasmodium falciparum infection, and anemia in children in the Gambia, West Africa. The American Journal of Tropical Medicine and Hygiene. 2010; 83: 965-972. DOI: 10.4269/ajtmh.2010.10-0311

[35] Nguyen HT, Tien TV, Tien NC, Ninh TU, Hoa NT. The effect of Olyset net screen to control the vector of dengue fever in Vietnam. Dengue Bulletin. 1996; 20: 87-92.

[36] Igarashi A. Impact of dengue virus infection and its control. FEMS Immunology \& Medical Microbiology. 1997; 18: 291-300. DOI: http://dx.doi.org/10.1111/j.1574-695X.1997. tb01058.x

[37] Hernández-Ávila JE, Rodriíguez M-H, Santos-Luna R, Sánchez-Castañeda V, RománPérezS, Ríos-Salgado VH, Salas-SarmientoJA. Nation-wide, web-based, geographicinformation system for the integrated surveillance and control of dengue fever in Mexico. PLOS One. 2013; 8(8): e70231. DOI: 10.1371/annotation/fa2d8273-9377-44c0-b61f-eb019beca2ce

[38] Vazquez-Prokopec GM, Galvin WA, Kelly R, Kitron U. A new, cost-effective, batterypowered aspirator for adult mosquito collections. Journal of Medical Entomology. 2009; 46: 1256-1259. DOI: http://dx.doi.org/10.1603/033.046.0602 
[39] González G, Ponce G, Quiroz-Martínez H, García JE, Flores AE. Insecticide resistance mechanisms in Aedes aegypti from Merida, Yucatan, Mexico, related with two dengue transmission seasons. Journal of American Mosquito Control Association. 2012; 28: 103.

[40] Ponce-García GP, Flores AE, Fernández-Salas I, Saavedra-Rodríguez K, Reyes-Solis G, Lozano-Fuentes S, Guillermo Bond J, Casas-Martínez M, Ramsey JM, García-Rejón J, Domínguez-Galera M, Ranson H, Hemingway J, Eisen L, Black IV WC. Recent rapid rise of a permethrin knock down resistance allele in Aedes aegypti in México. PLoS Neglected Tropical Diseases. 2009; 3(10): e531. DOI: 10.1371/journal.pntd.0000531

[41] Saavedra-Rodríguez K, Beaty M, Lozano-Fuentes S, Denham S, Garcia-Rejon J, ReyesSolis G, Machain-Williams C, Loroño-Pino MA, Flores-Suarez A, Ponce-Garcia G, Beaty B, Eisen L, Black IV WC. Local evolution of pyrethroid resistance offsets gene flow among Aedes aegypti collections in Yucatan State, Mexico. The American Society of Tropical Medicine and Hygiene. 2014; 92: 201-209. DOI: 10.4269/ajtmh.14-0277

[42] Flores-Suarez A, Ponce-Garcia G, Lopez-Monroy B, Villanueva-Segura O, RodriguezSanchez P, Arredondo-Jimenez JI, Manrique-Saide P. Current status of the insecticide resistance in Aedes aegypti (Diptera: Culicidae) from Mexico. In: Trdan S editor. Insecticides Resistance. InTech Editors; 2016. pp. 99-109. Croatia, EU. DOI: 10.5772/61526

[43] Hougard JM, Duchon S, Darriet F, Zaim M, Rogier C, Guillet P. Comparative performances, under laboratory conditions, of seven pyrethroid insecticides used for impregnation of mosquito nets. Bulletin of World Health Organization. 2003; 81: 324-333. DOI: https://dx.doi.org/10.1590/S0042-96862003000500006

[44] Jones C, Benítez-Valladares D, Barrera-Pérez M, Selem-Salas C, Chablé-Santos J, DzulManzanilla F, Che-Mendoza A, Guillermo-May G, Medina-Barreiro A, Sommerfeld J, Kroeger A, Manrique-Saide P. Use and acceptance of long lasting insecticidal nets for dengue prevention in Acapulco, Guerrero, Mexico. BMC Public Health. 2014; 14: 846. DOI: $10.1186 / 1471-2458-14-846$

[45] Vazquez-Prokopec G, Lenhart A, Manrique-Saide P. Housing improvement: a renewed paradigm for urban vector-borne disease control? Transactions of the Royal Society of Tropical Medicine and Hygiene. 2016; 110: 567-569. DOI: 10.1093/trstmh/trw070 
